\title{
'Chair-boat' transitions and side groups affect the stiffness of polysaccharides
}

\author{
Berthold Heymann, Helmut Grubmüller \\ Max-Planck-Institute for Biophysical Chemistry, Theoretical Molecular Biophysics Group, Am Faßberg 11, 37077 Göttingen, Germany
}

Received 23 November 1998; in final form 31 March 1999

\begin{abstract}
Atomic force microscopy experiments with individual $\beta$-(1,4)-glucose polymers (cellulose) and $\alpha$-(1,4)-glucose polymers (amylose) have been simulated by molecular dynamics stretching simulations, and force/extension curves were calculated. For forces above $1200 \mathrm{pN}$, we predict a stiffness of $5100 \mathrm{pN} / \AA$ for both polymers. In contrast to $\beta$-glucose, $\alpha$-glucose exhibits increased flexibility in the $300-1200 \mathrm{pN}$ range, which our simulations attribute to conformational 'chair-boat' transitions of the $\alpha$-glucose monomers. For $\beta$-glucose no such transitions were seen. We suggest a mechanism of neighbor inhibition, which should allow the tailoring of elastic properties through modification of inter-monomeric rotational flexibility, e.g., by introducing or removing bulky or charged side groups. (C) 1999 Elsevier Science B.V. All rights reserved.
\end{abstract}

\section{Introduction}

Recent advances in single-molecule atomic force microscopy (AFM) and optical tweezers techniques allow to study mechanical properties of individual molecules like the binding forces of protein-ligand complexes [1-6], the enforced unfolding of proteins [7-10], or the stiffness of polymers like DNA [11] or polysaccharides [12,13]. In these experiments, a force is applied to one end of a single molecule while the other end is kept fixed. Subsequently, the extension of that molecule as a function of the stretching force is recorded.

\footnotetext{
* Corresponding author. Fax: +49 551201 1089; e-mail: hgrubmu@gwdg.de
}

Computer simulations of such experiments [12,14-18] by means of molecular dynamics (MD) unbinding or stretching simulations can serve to predict the measured forces $[12,14]$ and are, therefore, now an established tool for complementing single-molecule AFM or optical tweezers experiments with microscopic interpretations in terms of molecular structures and interatomic interactions. In particular, single-molecule experiments and MD stretching simulations can explain macroscopic properties like stiffness at the molecular level.

Specifically, already relatively inexpensive in vacuo simulations of AFM experiments with polysaccharides (dextran) predicted three stiffness regions beyond the well-known entropic spring region at very weak forces, which were subsequently observed experimentally [12]: a region of moderate stiffness, followed by a transient region of high 
elasticity and a subsequent high stiffness region. Whereas the first of these three regions was already known from experiments with moderate forces, and was explained in terms of deformation of bond angles, the other two were unexpected. The simulations suggested conformational transitions of the sugar monomers to be the cause of the high flexibility region. The third region was attributed to bending of bond angles - similar to the first region, but stiffer, due to the changed geometry of the monomer.

In this Letter, we present force/extension curves calculated from MD simulations of the diastereomeric polysaccharides $\beta$ - $(1,4)$-glucose (cellulose), and $\alpha-(1,4)$-glucose (amylose). The elastic properties of these polysaccharides have been measured independently in single-molecule AFM experiments (see the Letter by $\mathrm{Li}$ et al. in this issue), which allows a direct comparison of simulation and experiment.

As can be seen in Fig. 2 (top row), the $D$-glucose polymers are structural isomers which differ only in the orientation of the linkage between the monomers: the $\mathrm{C} 1-\mathrm{O}$ bond is perpendicular to the ring plane for $\alpha$-glucose and within the plane for $\beta$-glucose. We ask to what extent the elastic properties of the polymers reflect this steric difference.

\section{Methods}

Molecular models for $\beta$ - and $\alpha$-16-mers in the chair conformation were generated using the molecular editor implemented in Quanta [19]. The two structures were minimized (steepest descent) until the gradient was less than $0.1 \mathrm{kcal} /(\mathrm{mol} \AA)$. For the minimization as well as for all subsequent simulations, force-field parameters and partial charges were taken from the CHARMM-based [20] Quanta library for polymer molecules with explicit treatment of all hydrogen atoms.

All MD simulations were carried out using the parallel MD program EGO [21], which implements efficient computations of Coulomb interactions using the 'fast multiple timestep structure adapted multipole' method [22], so that no electrostatic cut-off had to be used. The simulations were performed in vacuo and, in part, in explicit solvent (TIP3P water model [23]) using boundary conditions as described in Ref. [14]. An integration step size of 1 fs was used, and the length of chemical bonds to hydrogen atoms were fixed using the SHAKE algorithm [24]. The two initial structures were equilibrated for 120 ps by coupling to an external heat bath $(T=300 \mathrm{~K})$ using velocity rescaling [25] with a coupling constant of 10 $\mathrm{ps}^{-1}$. The resulting structures and velocities were used for the subsequent MD stretching simulations.

The top row of Fig. 2 shows part of these two structures (two monomers out of the total of 16) as well as the arrangement for the AFM simulations: The C4-bound terminal oxygen atom of the leftmost monomer was kept fixed at its initial position by a stiff harmonic potential with force constant $k_{0}=2.8$ $\mathrm{nN} / \AA$, while the C1-bound terminal oxygen atom to the right was subjected to a soft harmonic 'spring' potential (depicted as a spring in the figure) as described in Ref. [14] using the force constant $k=7$ $\mathrm{pN} / \AA$ of a typical AFM cantilever. In the course of AFM simulation, the spring potential was moved with constant velocity $v_{\text {pull }}$ towards the right, as indicated by the two arrows, thereby stretching the polymer. In these simulations, too, the system was coupled to a heat bath as described above.

The employed force field was checked against density functional calculations for an $\alpha-(1,4)$-glucose dimer (maltose). To that end, quasistatic 'AFM simulations' (i.e., as described above, but at $T=0 \mathrm{~K}$ ) were carried out using both the CHARMM force field as well as quantum mechanical energy minimization. For the latter calculations, we used the density functional program CPMD [26] with Vanderbilt pseudopotentials [27] in local-density approximation for the exchange correlation functional. For the plane wave expansion of the electron density we used an energy cutoff of 25 rydberg. CPMD was used through an interface implemented in EGO [28]. For both force-field calculations and quantum mechanical calculations, ring geometry and energies were recorded and compared.

A number of simulations addressed the influence of rotational flexibility between adjacent monomers. In these simulations, all $\Phi_{3}$ angles (as defined in the inset of Fig. 1) were constrained such that the ring planes of neighboring monomers were kept in an antiparallel orientation. This was achieved by including into the MD force field a restraining potential $U_{\text {restr }}:=U_{0} \cos \left(\Phi_{3}+\Delta \Phi\right)$ with the phase $\Delta \Phi=233^{\circ}$ chosen such that the antiparallel orientation $\left(\Phi_{3}=\right.$ 


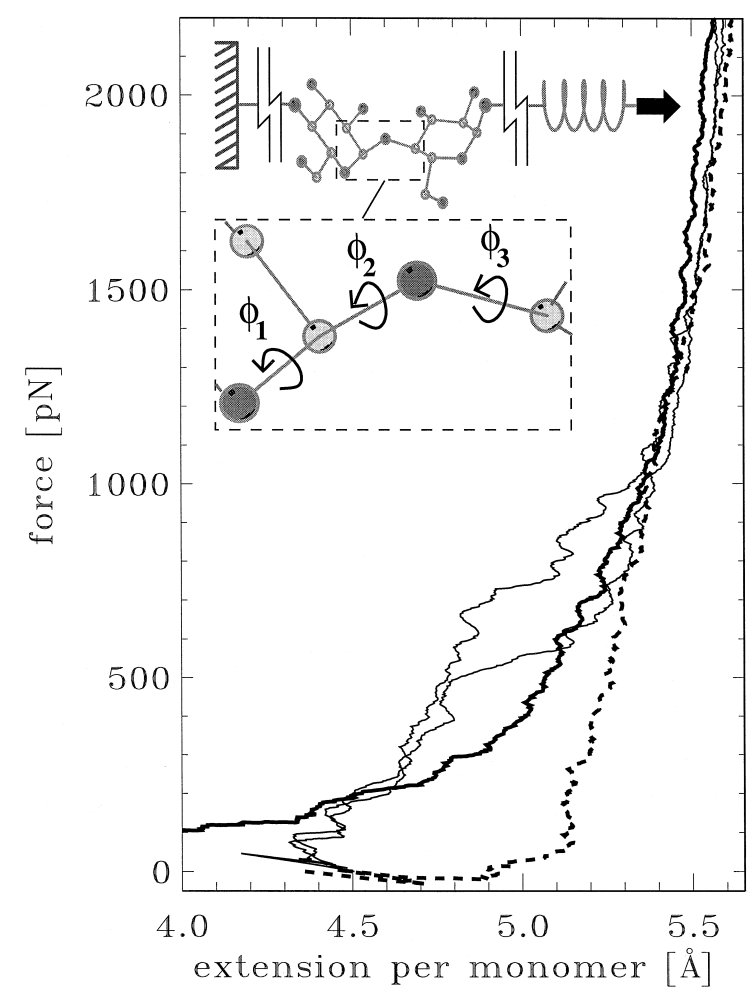

Fig. 1. Force/extension curves for $\beta$ - $(1,4)$-glucose (dashed curve) and $\alpha$ - $(1,4)$-glucose (three solid curves), derived from MD stretching simulations with $v_{\text {pull }}=10 \AA / \mathrm{ns}$. For $\alpha$ - $(1,4)$-glucose, simulations have been carried out with (thin curves) and without (bold curve) restraints for inter-monomeric rotations. Upper inset: arrangement of the simulation; only two of a total of 16 monomers are shown. Lower inset: Definition of the dihedral angles $\Phi_{1}, \Phi_{2}$ and $\Phi_{3}$, referred to in the text.

$-53^{\circ}$ ) was preferred. For the restraining strength $U_{0}$, we chose values between 2.5 and $10 \mathrm{kcal} / \mathrm{mol}$.

During the MD stretching simulations, all atomic coordinates as well as the applied pulling force were recorded every 100 fs. Additionally, the polymer extension was calculated from the distance between the terminal oxygens and recorded every femtosecond. Normalized force/extension curves were obtained by plotting the applied pulling force as a function of the polymer extension per monomer. The stiffness per monomer was derived from the slope of a linear fit to a suitable part of the force/extension curves, e.g. defined by a given force interval. Conformational changes were detected by monitoring the dihedral angles $\Phi_{1}$ and $\Phi_{2}$ (as defined in the insets of Fig. 1 as well as in Fig. 2, upper snapshots), which were also recorded every femtosecond.

To check if the obtained force/extension curves depend on the pulling velocity $v_{\text {pull }}$, a series of AFM simulations with $v_{\text {pull }}$ ranging from 500 down to 10 $\AA / n s$ was carried out. The most extended simulations with $v_{\text {pull }}=10 \AA / \mathrm{ns}$ covered $40 \mathrm{~ns}$ each; those were used for subsequent structure analysis.

\section{Results and discussion}

After equilibration, all monomers of the polymer remained in the chair conformation (shown in Fig. 2, top, for two out of the 16 monomers). No energy drifts were observed after 100 ps of the equilibration period.

Fig. 1 shows force/extension curves for $\beta-(1,4)$ glucose (dashed curve) and $\alpha-(1,4)$-glucose (three solid curves). For $\beta$-(1,4)-glucose, two regions can be distinguished: a high-elasticity region for forces below $100 \mathrm{pN}$, and a high-stiffness region for forces above $100 \mathrm{pN}$. The high-elasticity region is dominated by entropic forces of the polymer, which can be described well with the worm-like chain model [7] and shall not be analyzed further here. The high-stiffness region is apparently dominated by bond angle deformations. Closer inspection of the force/ extension curve in that region revealed a very slight shoulder (hardly visible in Fig. 1) with a stiffness between 3300 and $3600 \mathrm{pN} / \AA$ for stretching forces up to $1600 \mathrm{pN}$, and approximately $5100 \mathrm{pN} / \AA$ for larger pulling forces.

This stiffening of $\beta$-(1,4)-glucose is caused by tilts of the monomers along the polymer axis with respect to their respective neighbors, as illustrated by the two snapshots in the left half of Fig. 2. In the bottom snapshot, which we have chosen as a representative one, at a pulling force of $1700 \mathrm{pN}$ the right monomer has rotated with respect to the left monomer and around the bond marked ' $\Phi_{2}$ '. From inspection of the snapshot it might appear that this transition is dominated by a dihedral rotation around ' $\Phi_{3}$ '. However, by comparing the trajectories of $\Phi_{2}$ and $\Phi_{3}$, depicted in Fig. 2 (bottom left), one readily identifies $\Phi_{2}$ as the relevant dihedral angle. Accordingly, the inter-monomeric rotation was quantified by the $\Phi_{2}$ 

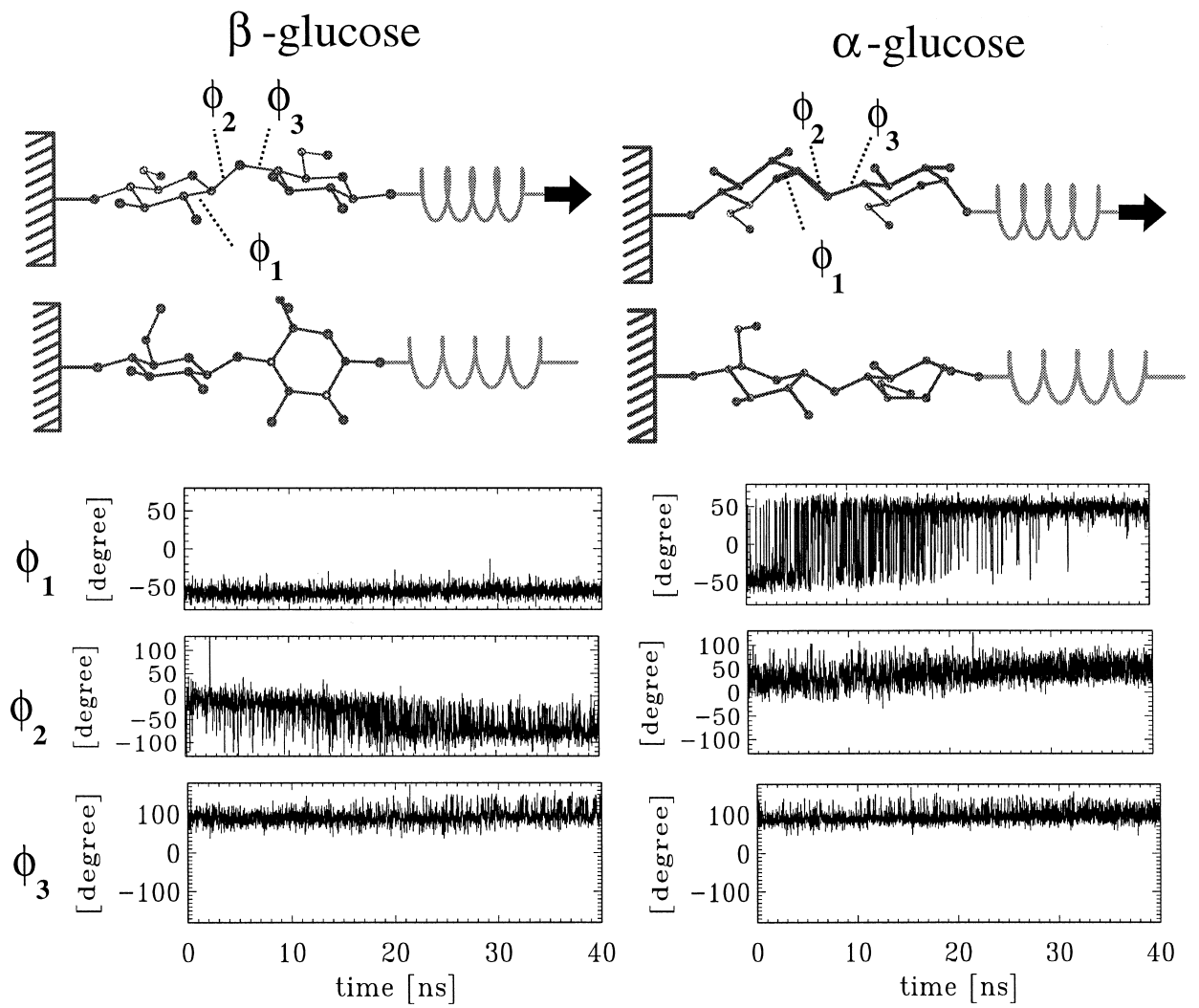

Fig. 2. Conformational changes in $\beta$-(1,4)-glucose (left) and in $\alpha$-(1,4)-glucose (right) during the MD stretching simulations. The upper structures show the initial conformations of the two polymers (only two out of the 16 monomers are shown); the lower structures show representative snapshots after $30 \mathrm{~ns}$, at a stretching force of $1700 \mathrm{pN}$. The development of the three dihedral angles, $\Phi_{1}, \Phi_{2}$, and $\Phi_{3}$, as defined in the top structures (cf. also Fig. 1) during the simulations is shown below.

trace, which displays transitions of $\Phi_{2}$ from its initial value of $0^{\circ}$ to a final value of $-60^{\circ}$. These transitions are observed mainly between 15 and 22 ns, corresponding to pulling forces of 1300-1600 pN. Since this tilt entails only a very small polymer elongation of less than $0.1 \AA$ per monomer, its fingerprint in the force/extension curve is only dim.

In contrast, the force/extension curve of $\alpha$ (1,4)-glucose (Fig. 1; solid, bold line) exhibits a much higher elasticity in the force range between 250 and $1000 \mathrm{pN}$. (The two thin lines are discussed below.) The snapshots in Fig. 2 (right) reveal a chair-boat transition within each of the monomers. This intra-monomeric conformational transition involves an elongation of approximately $0.5 \AA$ per monomer - much larger than for $\beta$ - $(1,4)$-glucose. Note also that the $\alpha$-glucose monomers did not change their orientation, as was the case for the $\beta$-(1,4)-glucose (cf. the $\Phi_{2}$ traces at the bottom).

The dihedral angle $\Phi_{1}$ (definition: see upper snapshots or Fig. 1) was selected as a probe for these chair-boat transitions. In the respective trace (upper curve) the chair conformation is characterized by $\Phi_{1} \approx-50^{\circ}$, and the boat conformation by $\Phi_{1} \approx 50^{\circ}$. The frequent jumps of $\Phi_{1}$ reflect a large number of these force-induced transitions for all monomers, however spread out over a large time (and, hence, force) interval. Already at very low forces the monomers started to show increasingly frequent conformational transitions until, after $25 \mathrm{~ns}$ and at a pulling force of ca. $1000 \mathrm{pN}$, nearly all monomers have adopted the boat conformation. Apparently, each of the monomers underwent many chair-boat flips back and forth. 
For transitions which are independent from each other, most transitions would occur collectively near a critical pulling force sufficiently large to overcome the transition barrier. Because the chair-boat transitions involve a significant elongation of the monomer, such collective transitions would entail a rapid elongation of the entire polymer within a relatively small force interval, thereby yielding a shoulder in the force/extension curve.

Since in our simulation no such shoulder, but, instead, a wide transition range was observed, we performed a correlation analysis for transition events [29]. That analysis, indeed, revealed significant anticorrelations for the transitions of adjacent monomers: whereas, initially, transitions of insular monomers could be induced with relatively small forces when both of their neighbors were still in chair conformation, much larger forces appeared to be necessary later, when one or both neighbors had already adopted the boat conformation. Apparently, the first few insular transitions hindered subsequent transitions of adjacent monomers, which explains why increasingly larger forces were required to drive further transitions. This 'anti-cooperativity' suppressed the expected collectivity of the transitions, and, therefore, no shoulder was observed in our simulations.

These results were at variance with experimental AFM force/extension curves for $\alpha$ - $(1,4)$-glucose at intermediate forces, however, which did show a pronounced shoulder (see the Letter by $\mathrm{Li}$ et al. in this issue). That shoulder appeared quite similar to the one observed for dextran [12] which there was interpreted in terms of collective inter-monomeric conformational transitions. Accordingly, for the dextran simulations, no anti-correlations were seen. What is the reason for the anti-correlations observed here in our simulations of $\alpha$ - $(1,4)$-glucose?

A closer analysis [29] suggested that parallel orientations of the ring planes are necessary for the anti-correlations to occur. Indeed, in all of our MD stretching simulations, the ring planes adopted parallel orientation, even if their orientation was initially set at random.

To check if this ubiquitous parallel ring plane alignment is an artifact of our in vacuo simulations, the simulations were repeated in water solvent as described in Section 2. No significant deviations from the in vacuo simulations were observed. In particular, the monomers still aligned in parallel orientation, and the force/extension curves were essentially unchanged (data not shown).

To check the employed force field, density functional calculations on an $\alpha$ - $(1,4)$-glucose dimer were carried out and compared to force-field calculations as described in Section 2. As shown in Fig. 3, the results obtained from the quantum mechanical calculations (symbols) showed good agreement with the results from the force field calculations (lines): probed by $\Phi_{1}$ (upper picture), the chair-boat transition occurred at nearly identical polymer extensions, and also for the (minimum) total energies as a function of extension the CHARMM force field (lower graph, lines) agreed well with the density functional values (symbols).
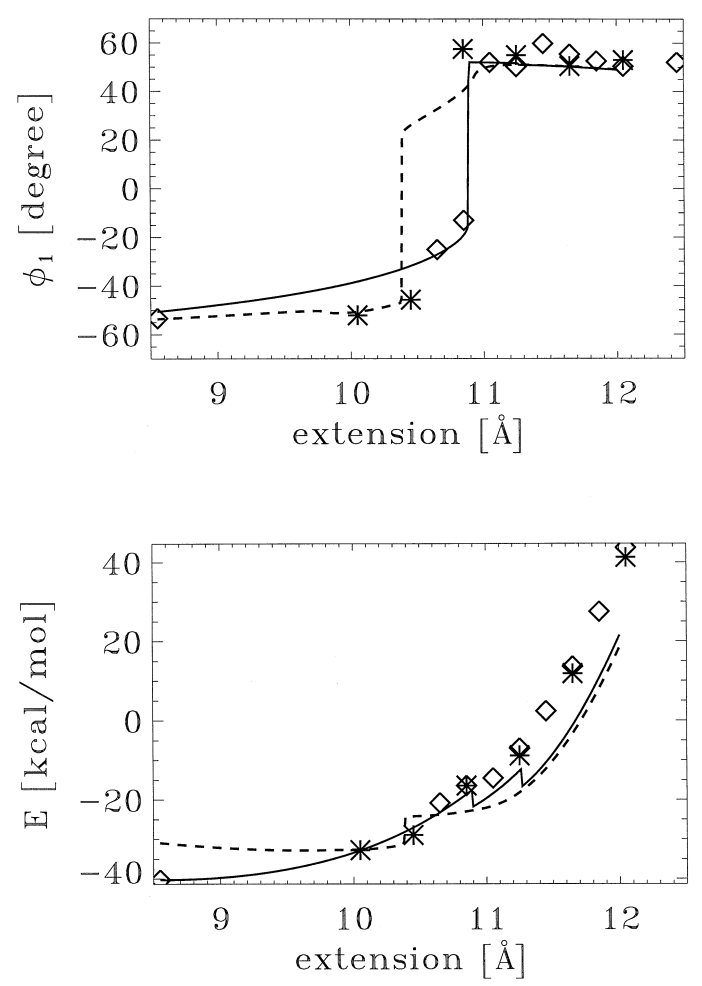

Fig. 3. Comparison of the employed CHARMM force field (lines) with density functional calculations (symbols) during stretching (solid lines and diamonds) and subsequent release (dashed lines and stars) of an $\alpha$-(1,4)-glucose dimer. Upper graph: dihedral angle $\Phi_{1}$ reflecting a chair-boat transition; lower graph: potential (lines) and ground state energy (symbols), the slope of which defines the stretching force. 
Assuming that the parallel alignment of adjacent monomers is indeed suppressed in the experiment, these findings led us to propose that the polymers actually used in the AFM experiment differ from the $\alpha$-glucose polymer modeled on the basis of the information we had (Fig. 2, top right). In particular, bulky or charged side groups can prevent parallel monomer alignment either sterically or energetically and thereby weaken the neighbor inhibition. Indeed, protection groups are often used to solubilize or neutralize polysaccharides.

To test our hypothesis, we included the primary steric effect of bulky side groups by weakly restraining the ring plane orientation of neighboring monomers to prefer antiparallel orientation, as described in Section 2. As expected, the resulting force/extension curves in Fig. 1 (thin, solid lines) now developed pronounced shoulders, which were absent without orientation restraint (bold line). Furthermore, the forces at which the shoulders occurred varied with the strength $U_{0}$ of the restraining potential. The upper and lower thin traces show as two examples force/extension curves for restraining strengths of $U_{0}=5$ and $U_{0}=3.75 \mathrm{kcal} / \mathrm{mol}$, respectively.

\section{Summary and conclusion}

We have presented a series of MD stretching simulations of the diastereomeric polysaccharides $\beta$-(1,4)-glucose and $\alpha$-(1,4)-glucose, from which we have computed their elastic properties.

Our simulations explained these properties at the atomic level in terms of conformational transitions. Despite the structural similarity of the two polymers, these transitions differed significantly from each other with respect to the underlying microscopic processes as well as to their effect on polymer stiffness. Stretching of $\beta$ - $(1,4)$-glucose induced only slight, inter-monomeric rotations that did not significantly affect its elastic properties; in contrast, $\alpha$ - $(1,4)$-glucose exhibited large intra-monomeric chair-boat transitions which led to a continuous stiffening of the molecule for pulling forces between 250 and 1000 $\mathrm{pN}$. These transitions exhibited strong anti-cooperativity through next-neighbor inhibition.

Further MD stretching simulations showed, however, that this anti-cooperativity occurred only if the ring planes of adjacent monomers are oriented in parallel. As the monomers were restrained to antiparallel orientation, no anti-cooperativity was observed and, therefore, the chair-boat transitions occurred almost collectively. In that case, pronounced shoulders in the force/extension curve appeared. This result suggests that shoulders in force/extension curves can be caused by bulky or charged side groups, steric or energetic hindrance of which renders parallel alignment of the monomers unfavorable.

Moreover, for different strengths of the restraining torsion potential our simulations showed shoulders at different forces. From the available data, the particular force at which the shoulder should occur in the AFM experiments could thus not be determined; such a prediction would require the inclusion of an atomic model of the putative side group, which was impractical due to the large number of potential candidates.

Our simulations displayed a remarkable sensitivity of elastic properties to slight microscopic modifications. Examples studied here in detail are the effects of monomer linkage modifications and of inter-monomeric rotational flexibility on force/extension curves. In particular, the observed anti-cooperativity implies an unexpected mechanism that could be utilized to tailor the elastic properties of polymers. Accordingly, modification of side groups should allow the shaping of the force/extension curves by adjusting inter-monomeric rotational flexibility.

\section{Acknowledgements}

We thank Hermann Gaub and Hongbin Li for discussing experimental details and for providing their amylose results prior to publication, Markus Eichinger for help with the density functional calculations, and Paul Tavan for suggesting the density functional calculations. Density functional calculations were carried out on the Cray T3E at the Rechenzentrum der Universität Stuttgart, Germany, project 11392. This work was supported by the Deutsche Forschungsgemeinschaft, Grant GR 1590/1-1. 


\section{References}

[1] G.U. Lee, D.A. Kidwell, R.J. Colton, Langmuir 10 (1994) 354.

[2] E.-L. Florin, V.T. Moy, H.E. Gaub, Science 264 (1994) 415.

[3] V.T. Moy, E.-L. Florin, H.E. Gaub, Science 266 (1995) 257.

[4] P. Hinterdorfer, W. Baumgartner, H.J. Gruber, K. Schlicher, H. Schindler, Proc. Natl. Acad. Sci. USA 93 (1996) 3477.

[5] S. Allen, X. Chen, J. Davies, M.C. Davies, A.C. Dawkes, J.C. Edwards, C.J. Roberts, J. Sefton, S.J.B. Tendler, P.M. Williams, Biochemistry 36 (1997) 7457.

[6] R. Ros, F. Schwesinger, D. Anselmetti, M. Kubon, R. Schäfer, A. Plückthun, L. Tiefenauer, Proc. Natl. Acad. Sci. USA 95 (1998) 7402.

[7] M. Rief, M. Gautel, F. Oesterhelt, J.M. Fernandez, H.E. Gaub, Science 276 (1997) 1109.

[8] M.S.Z. Kellermayer, S.B. Smith, H.L. Granzier, C. Bustamante, Science 276 (1997) 1112.

[9] L. Tskhovrebova, J. Trinck, J.A. Sleep, R.M. Simmons, Nature 387 (1997) 308.

[10] A.F. Oberhauser, P.E. Marszalek, H.P. Erickson, J.M. Fernandez, Nature 393 (1998) 181.

[11] G.U. Lee, L.A. Chrisey, R.J. Colton, Science 266 (1994) 771.

[12] M. Rief, F. Oesterhelt, B. Heymann, H.E. Gaub, Science 275 (1997) 1295.

[13] H. Li, M. Rief, F. Oesterhelt, H.E. Gaub, Adv. Materials 3 (1998) 316.

[14] H. Grubmüller, B. Heymann, P. Tavan, Science 271 (1996) 997.
[15] M.W. Konrad, J.I. Bolonick, J. Am. Chem. Soc. 118 (1996) 10986.

[16] S. Izrailev, S. Stepaniants, M. Balsera, Y. Oono, K. Schulten, Biophys. J. 72 (1997) 1568.

[17] S. Marrink, O. Berger, P. Tieleman, F. Jähnig, Biophys. J. 74 (1998) 931

[18] H. Lu, B. Isralewitz, A. Krammer, V. Vogel, K. Schulten, Biophys. J. 75 (1998) 662.

[19] Quanta97, Molecular Simulations, Inc., University of York, York, UK, 1986-1998.

[20] C.L. Brooks III, M. Karplus, J. Chem. Phys. 79 (1983) 6312.

[21] M. Eichinger, H. Grubmüller, H. Heller, User Manual for EGO_VIII, Release 2.0, electronic access: http:// www.mpibpc.gwdg.de/abteilungen/071/ego.html.

[22] M. Eichinger, H. Grubmüller, H. Heller, P. Tavan, J. Comput. Chem. 18 (1997) 1729.

[23] W.L. Jorgensen, J. Chandrasekhar, J.D. Madura, R.W. Impey, M.L. Klein, J. Phys. Chem. 79 (1983) 926.

[24] J.P. Ryckaert, G. Ciccotti, H.J.C. Berendsen, J. Comput. Phys. 23 (1977) 327.

[25] H.J.C. Berendsen, J.P.M. Postma, W.F. van Gunsteren, A. Dinola, J.R. Haak, J. Chem. Phys. 81 (1984) 3684.

[26] J. Hutter, P. Ballone, M. Bernasconi, P. Focher, E. Fois, St. Goedecker, D. Marx, M. Parrinello, M. Tuckerman, CPMD Version 3.0, MPI für Festkörperforschung and IBM Zurich Research Laboratory 1995-1996.

[27] D. Vanderbilt, Phys. Rev. B 41 (1990) 7892.

[28] M. Eichinger et al., submitted.

[29] B. Heymann, H. Grubmüller, in preparation. 